\title{
Prevenção de recaídas baseada em mindfulness no tratamento do tabagismo - Brasil*
}

\section{Relapse Prevention Based on Mindfulness in the Treatment of Tobacco Dependence - Brazil}

\section{Prevención de recaídas basada en Mindfulness en el tratamiento del tabaquismo - Brasil}

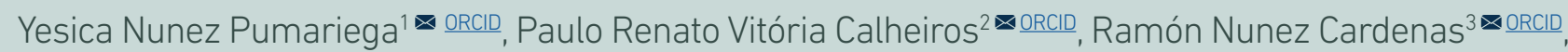
Edson dos Santos Farias $\underline{\text { ORCID }}$, Catalina Dominga Pumariega Torres 5

${ }^{1}$ Universidade Federal de Rondônia

Brasil

\section{Fecha correspondencia:} Recibido: agosto 1 de 2019. Aceptado: abril 14 de 2020.

\section{Forma de citar:}

Nunez Pumariega, Y., Vitória Calheiros, P. R., Nunez Cardenas, R., Farias, E.S., \& Pumariega Torres, C. D. (2020). Prevenção de recaídas baseada em mindfulness no tratamento do tabagismo - Brasil. Rev. CES Psico, 13(2), 129-143.

\section{Open access}

(c) Copyright

Licencia creative commons

Etica de publicaciones

Revisión por pares

Gestión por Open Journal System DOI: http://dx.doi.org/10.21615/ cesp.13.2.9

ISSN: 2011-3080

\section{Resumo}

Realizou-se um ensaio clínico randomizado com o objetivo de analisar os efeitos de um programa de Prevenção de Recaídas baseado em mindfulness no tratamento do tabagismo. 0 estudo foi conduzido no Centro de Atenção Psicossocial de Álcool e Drogas (CAPSad) em 2017 na cidade de Porto Velho, capital de Rondônia, Brasil. Participaram da pesquisa 52 pacientes em tratamento para o tabagismo, com idades entre 29 a 60 anos de ambos os sexos, usuários apenas de tabaco. Para a realização deste trabalho foram formados dois grupos: $\mathrm{G} 1$ =experimental e $\mathrm{G} 2=$ controle, ambos formados por 26 cada um. No pré-teste, os dados mostraram não haver diferença significativa entre esses dois grupos. Na comparação do pós-teste ocorreu uma diferença significativa na diminuição dos sintomas de ansiedade $(p=0,043)$ e nível de monóxido de carbono-CO2 $(p=0,03)$ nos pulmões dos participantes. No resultado da análise de proporção, houve diferença no G1 pós-intervenção com diminuição de sintomas de ansiedade ( $p=<0,001$ ), depressão ( $p=0,012)$ e monóxido de carbono ( $p=<0,001)$, respectivamente. Estes achados mostraram efeitos significativos do mindfulness para a prevenção de recaídas e diminuição de sintomas de ansiedade, depressão e nível de monóxido de carbono nos pulmões, no G1 pós-intervenção nos pacientes fumantes e fumantes dependentes em tratamento no CAPSad.

Palavras-chave: Tabagismo, Mindfulness, Depressão, Ansiedade, Monóxido de Carbono, Comportamento Viciante.

\section{Abstract}

A randomized clinical trial was conducted to analyze the effects of a Mindfulness-based relapse prevention program for tobacco dependence treatment. The study was conducted at the Center for Psychosocial Care of Alcohol and Drugs (CAPSad), in the city of Porto Velho, capital of Rondônia, Brazil, 2017. 52 patients aged between 29 to 60, of both sexes, who were only 


\section{Sobre el artículo:}

${ }^{*}$ Agradecimentos: Ao Centro de Atenção Psicossocial de Álcool e Drogas (CAPSad) da cidade de Porto Velho, que proporcionou os prontuários e a participação de pacientes registrados neste Centro para o tratamento do tabagismo.

\section{Sobre los autores:}

1. Mestre em Psicologia pela Universidade Federal de Rondônia-Brasil. Especialista em Terapia Cognitivo Comportamental.

2. Doutor em Psicologia. Professor do Departamento de Psicologia da Universidade Federal de Rondônia-Brasil.

\section{Doutor em Biologia} Experimental. Professor do Departamento de Educação Física da Universidade Federal de Rondônia-Brasil. Líder do grupo de pesquisa em psicologia do Exercício Físico e Esporte na promoção da Saúde.

4. Doutorado em Saúde da Criança e Adolescente. Professor do Departamento de Educação Física da Universidade Federal de Rondônia-Brasil.

5. Pedagoga e Especialista em Supervisão Escolar pela Faculdade de Porto Getúlio Vargas-Rondônia-Brasil. tobacco users, participated in the research. To carry out this study, two groups were made up: $G 1$ = experimental and $G 2$ = control, both consisted of 26 people. In the pre-test, the data showed that there was no significant difference between these two groups. In the post-test comparison, there was a considerable difference in the reduction of anxiety symptoms ( $p=0.043)$ and carbon monoxide-CO2 level $(p=0.03)$ in the lungs of smoking patients. In the result of the proportion analysis, there was a difference in $G 1$ post-intervention with decreased symptoms of anxiety $(p=<0.001)$, depression ( $p=0.012$ ) and carbon monoxide ( $p=<0.001)$, respectively. These findings showed significant effects of mindfulness in preventing relapse and reducing symptoms of anxiety, depression and carbon monoxide level the post-intervention group of smokers and dependent smokers treated at CAPSad.

Keyword: Smoking, Tobacco Dependence, Mindfulness, Depression, Anxiety, Carbon Monoxide, Addictive Behavior.

\section{Resumen}

Se realizó un ensayo clínico aleatorizado con el objetivo de analizar los efectos de un programa de prevención de recaídas basado en Mindfulness para el tratamiento del tabaquismo. El estudio se realizó en el Centro de Atención Psicosocial del Alcohol y las Drogas (CAPSad), en 2017, en la ciudad de Porto Velho, capital de Rondônia, Brasil. Participaron en la investigación 52 pacientes, de ambos sexos, con edades comprendidas entre 29 y 60 años, consumidores de tabaco solamente. Para la realización de este trabajo, se formaron dos grupos: G1 = experimental y G2 = control, formados por 26 personas cada uno. En la prueba previa, los datos mostraron que no había diferencia significativa entre los grupos. En la comparación de los resultados en la prueba posterior se halló una diferencia significativa en la reducción de los síntomas de ansiedad ( $p=0.043)$ y en el nível de monóxido de carbono-CO2 ( $p=0.03)$ em los pulmones de los participantes. Como resultado del análisis de proporciones, se presentó una diferencia en el $\mathrm{G} 1$ en la prueba posterior, con síntomas disminuidos de ansiedad $(p=<0.001)$, depresión $(p=0.012)$ y monóxido de carbono $(<0.001)$, respectivamente. Estos hallazgos mostraron los efectos significativos del mindfulness para la prevención de recaídas y reducción de los síntomas de ansiedad, depresión y nível de monóxido de carbono em los pulmones, en el grupo de experimento posterior a la intervención en fumadores y fumadores dependientes que reciben tratamiento en el CAPSad.

Palabra clave: Tabaquismo, Mindfulness, Depresión, Ansiedad, Monóxido de Carbono, Comportamiento Adictivo.

\section{Introdução}

O Tabagismo tem sido um tema de interesse científico nas últimas décadas, há um amplo consenso na literatura que a reconhece como uma doença resultante da dependência à nicotina, constituindo-a como a segunda causa de morte evitável no mundo, sendo considerado um grave problema de saúde pública (Nunes \& Castro, 2010; Diehl, Cordeiro, \& Laranjeira, 2011). Estima-se que, no Brasil, 11 milhões de mulheres e 16 milhões de homens sejam fumantes, concentrando-se na faixa etária dos 20 aos 49 anos de idade (Instituto Nacional do Câncer (INCA), 2017).

O tabagismo faz parte das 10 primeiras causas de mortes no mundo, provocando enfermidades como: doenças cardíacas isquêmicas, acidente vascular cerebral, doença pulmonar obstrutiva crônica (DPOC), infecções do trato respiratório inferior e o câncer de pulmão ou traqueia (World Health Organization, 2018). 
Pág 131

Atualmente existe uma variedade considerável de evidências científicas sobre os benefícios de mindfulness no bem-estar físico e psíquico daqueles que a praticam (Weiss \& Noto, 2017). Apesar de ser uma técnica psicoterápica relativamente nova e pouco praticada no Brasil, estudos em países como Estados Unidos têm demonstrado que se trata de uma prática aceitável de implantação e implementação para o tratamento de recaída, mostrando resultados positivos e promissores para a prevenção e cessação no controle da dependência do tabaco (Weiss \& Noto, 2017).
Embora o Brasil tenha conseguido nos últimos 25 anos diminuir a porcentagem de fumantes de $29 \%$ para $12 \%$, ainda ocupa o oitavo lugar no número de fumantes no mundo (Reitsma \& Fullman, 2017). Os dados sobre a redução significativa de consumo se confirmaram também no estudo realizado pelo INCA nas capitais brasileiras em 2017; indicando a prevalência de tabagismo no Brasil em 10,5\%.

Entre os fatores que dificultam o objetivo de parar de fumar estão: motivação para a mudança de comportamento, a síndrome de abstinência e as comorbidades psiquiátricas (Rondinao, Gorayeb, \& Botelho, 2007; Diehl et al., 2011). Muitos fatores podem estar envolvidos numa recaída, entre eles, conflitos interpessoais e problemas comórbidos, como a ansiedade e a depressão (Marlatt \& Donovan, 2009). Os métodos de tratamento preconizados como primeira linha são: a terapia de reposição de nicotina, a utilização de bupropiona e a terapia cognitiva comportamental (Marques, 2001).

Entre as intervenções cognitivos comportamentais aplicadas ao tratamento psicoterápico do tabagismo pode-se citar o Modelo Transitório de Mudança Intencional de Comportamento (Prochaska \& DiClementi, 1982), a Entrevista Motivacional (Miller \& Rollnick, 2001), a Prevenção de Recaídas (Marlatt \& Donovan, 2009) e a Psicoterapia Cognitivo Comportamental (Beck, 2013). Recentemente um modelo novo de psicoterapia baseada no construto de mindfulness apresentou novas possiblidades.

0 mindfulness é praticado no oriente há mais de 2.500 anos. As abordagens da Terapia Cognitivo Comportamental passaram a incorporá-la a partir dos anos 1990 (Wenzel. Brown, \& Beck, 2010). Esta técnica, apesar de ser antiga tem se tornado uma forte tendência entre as psicoterapias das últimas décadas. Esta prática é compreendida como uma habilidade metacognitiva, que consiste em manter a atenção plena no momento presente, e tem como pretensão, ampliar a consciência dos indivíduos sobre seus pensamentos, emoções e sentimentos.

Atualmente existe uma variedade considerável de evidências científicas sobre os benefícios desta prática no bem-estar físico e psíquico daqueles que a praticam (Weiss \& Noto, 2017). Apesar de ser uma técnica psicoterápica relativamente nova e pouco praticada no Brasil, estudos em países como Estados Unidos têm demonstrado que se trata de uma prática aceitável de implantação e implementação para o tratamento de recaída, mostrando resultados positivos e promissores para a prevenção e cessação no controle da dependência do tabaco (Weiss \& Noto, 2017).

Davis et al. (2014) realizaram um estudo randomizado comparando um programa de tratamento para parar de fumar, Mindfulness Training for Smokers (MTS) com uma terapia de cuidado habitual (Controle), que incluiu a disponibilidade de uma linha telefônica para parar de fumar e adesivos de nicotina. Os dados foram coletados de 198 fumantes de baixo nível socioeconômico. 0 desfecho primário foi a abstinência do tabagismo por seis meses, medida por testes respiratórios com monóxido de carbono.

Os resultados desses estudos sugerem que o mindfulness pode ser um relevante recurso aplicado ao tratamento do tabagismo. Em virtude disso, este estudo procurou analisar os efeitos de um programa de prevenção de recaída baseado em mindfulness aplicada ao tratamento do tabagismo. De tal modo, este trabalho coloca-se como um elemento a mais no processo de junção de esforços na avaliação de novos recursos para o tratamento do tabagismo. 
Foi realizado um ensaio clínico randomizado desenvolvido no período de um ano (12 meses), no Centro de Atenção Psicossocial de Álcool e Drogas (CAPSad) do Município de Porto Velho capital do Estado de Rondônia - Brasil.

\section{Método}

\section{Característica do Estudo}

Foi realizado um ensaio clínico randomizado desenvolvido no período de um ano (12 meses), no Centro de Atenção Psicossocial de Álcool e Drogas (CAPSad) do Município de Porto Velho - capital do Estado de Rondônia - Brasil. Esta pesquisa foi aprovada pelo comitê de ética em pesquisa da Universidade Federal de Rondônia através do parecer de número 1.849.754 e CAAE 60727716.0.0000.5300 CEP/CONEP.

\section{População}

A população do estudo foi composta por 60 pacientes em tratamento para o tabagismo no CAPSad no Município de Porto Velho- R0, realizado no ano de 2017.

0 cálculo do tamanho da amostra foi realizado através do programa Statcal (Epiinfo). Baseou-se na estimativa de $50 \%$ de indivíduos em tratamento de recaída do tabagismo com erro amostral de cinco pontos percentuais (5\%) e Intervalo de Confiança de $95 \%$ $\left(\mathrm{IC}_{95 \%}\right)$. A amostra foi dividida em dois grupos $\mathrm{G} 1=$ Experimental e $\mathrm{G} 2=$ Controle, para 0 pareamento dos grupos foi utilizada uma randomização simples, grupo experimental $=26$ e controle $=26$, totalizando 52 pacientes.

\section{Instrumentos}

Para alcançar os objetivos deste estudo contou-se com cinco instrumentos a seguir:

Questionário sociodemográfico

Teve como pretensão conhecer as características individuais dos pacientes, por meio das variáveis: idade, gênero, estado civil, classe socioeconômica (ABEP, 2017) e grau de escolaridade. Para a classificação da classe econômica das famílias, utilizou-se a classificação da Associação Brasileira de Empresas de Pesquisa (ABEP). 0 critério da ABEP é um instrumento de segmentação econômica que utiliza o levantamento de características domiciliares (presença e quantidade de alguns itens domiciliares de conforto e grau de escolaridade do chefe de família) para diferenciar a população. 0 critério atribui pontos em função de cada característica domiciliar e realiza a soma dos mesmos; a partir disso, é feita a correspondência entre faixas de pontuação do critério e estratos de classificação econômica definida por classe alta: A (A1-A2), média alta:(B1-B2), média baixa: C (C1-C2), baixa: D e E.

\section{Teste de Fagerström}

O teste tem o objetivo de estimar o grau de dependência nicotínica, este instrumento é mundialmente utilizado como ferramenta de avaliação, em substituição a outros testes altamente custosos e que consomem mais tempo de aplicação. Foi introduzido por Fagerstrõm (1989), como questionário de tolerância de Fagerström (FTQ), sendo validado e adaptado no Brasil por Carmo e Pueyo (2002). Com isso, este passou a se chamar teste de dependência à nicotina (Pietrobon, Barbisan, \& Manfroi, 2007). 0 grau de dependência nicotínica é avaliado ao final segundo o resultado da soma, muito baixo (0 a 2), baixo (3 a 4), média (5), elevada (6-7) e muito elevada (8 a 10). A confiabilidade da consistência interna calculada pelo coeficiente a de alfa - Cronbach, teve um valor de 0,642, oscilando no entorno de 0,60 e 0,70, mesmo assim, a correlação teste-reteste mostrou um valor de 0,915. Reconhecendo o instrumento um alto nível de confiabilidade e validade para aplicações no contexto clínico do tabagismo (Carmo \& Pueyo, 2002). 


\begin{abstract}
Monoxímetro
Utilizou-se este aparelho para detectar o tipo de fumante e o nível de monóxido de carbono nos pulmões dos pacientes tabagistas. 0 monoxímetro ou também chamado de bafômetro do cigarro, é um aparelho que mede a concentração de monóxido de carbono no organismo do fumante. Os resultados aparecem no visor do aparelho após o fumante assoprar pelo bocal descartável do equipamento. Depois disso, luzes se acendem e um sinal sonoro indica o resultado final do teste. Desta forma, obteve-se o dado da concentração de monóxido de carbono (CO2), seguindo o previsto de caracterização na tabela $\mathrm{COHb}$ bedfont scientific contributions to health, sendo considerados $<1,5 \%$ não fumantes; $\geq 1,5 \%$ e $\leq 2 \%$ zona de perigo e $>2 \%$ dependência à nicotina (Carmo \& Pueyo, 2002).
\end{abstract}

Inventário de Depressão de Beck (BDI) (Beck et al., 1961)

Trata-se de um recurso projetado para registrar a presença e a intensidade de sintomas depressivos. A atual versão BDI-II, foi validada por Gomes-Oliveira, Gorenstein, Lotufo Neto, Andrade e Wang (2012), sendo considerada por especialistas um dos instrumentos mais utilizados e uma das melhores medidas de triagem da depressão (Gorenstein \& Wang, 2016). Este questionário consiste em 21 grupos de afirmações. 0 avaliado assina o item próximo a afirmação que descreve a melhor maneira como ele se sentiu na última semana, incluindo o dia da aplicação do inventário. A pontuação final indica a intensidade dos sintomas de 0 a 13: depressão mínima, 14 a 19: depressão leve, 20 a 28: depressão moderada e 29 a 63: depressão severa. 0 coeficiente alfa de Cronbach foi 0,92. A confiabilidade teste-reteste foi de 9,84 (DP 10,65). 0 ponto de corte de 10/11 no BDI-II para detectar a depressão maior apresentou uma sensibilidade de $70 \%$ e especificidade de $84,4 \%$. A correlação do BDI-II com SRQ-20 foi 0,849, K10 0,846, MADRS 0,748 e HAM-A 0,663. O BDI-II discrimina corretamente 81,1\% dos casos de depressão ausente/mínima (0-13) e 85,7\% dos casos de depressão grave (29-63). Após sua validação mostrou ser um instrumento homogêneo e estável com evidências de validade de critério, concorrente e discriminante.

Inventário de Ansiedade de Beck/Beck Anxiety Inventory (BAI)

Esse instrumento consiste em uma escala de autorrelato desenvolvido para medir a intensidade de sintomas de ansiedade. Foram selecionados 21 itens que refletissem, somaticamente, afetivamente e cognitivamente, os sintomas das características de ansiedade (Beck et al., 1988; Cunha, 2001). Ao respondente, é perguntado o quanto ele (a) sente-se incomodado, por cada um dos sintomas, durante a semana que passou, dentro de uma escala do tipo Likert de 4 pontos, que refletem níveis de gravidade crescente de cada sintoma variando de 0 a 3: 1) absolutamente não; 2) levemente: não me incomodou muito; 3) moderadamente: foi muito desagradável, mas pode suportar; 4) gravemente: dificilmente pode suportar (Beck et al., 1988, Beck \& Steer. 1993; Cunha, 2001). 0 escore total é o resultado da soma dos escores dos itens individuais e pode variar de 0 a 63, a maior pontuação corresponde a um grau mais elevado de ansiedade. As indicações para interpretar a ansiedade auto informada, de acordo com a adaptação brasileira, são os seguintes pontos de corte: 0 a 10: ansiedade mínima; 11 a 19: ansiedade leve; 20 a 30: ansiedade moderada; 31 a 63: ansiedade grave. No Brasil, foi validado por Cunha (2001). Sua estimativa de fidedignidade, baseada no Coeficiente Alfa de Cronbach do BAl foi de 0.88, o que pode ser interpretado como satisfatório, demonstrando que o instrumento possui um bom nível de precisão para medir intensidade de depressão. 
Pág 134

No G1 utilizou-se uma adaptação do programa de Prevenção de Recaídas baseada em mindfulness para comportamento de recaídas (Bowen, Chawla, \& Marlatt, 2015). Foi desenvolvido em oito sessões, com encontros semanais, tendo uma durabilidade de uma hora e meia a duas horas no máximo.

\section{Procedimentos}

No primeiro momento, foi estabelecido contato com a equipe do CAPSad para conhecer o ambiente físico, a equipe de profissionais e entender como funcionava a dinâmica da instituição, assim como, conhecer os procedimentos do tratamento oferecido aos pacientes tabagistas.

Em seguida, foi apresentado o projeto à direção do CAPSad e a Secretaria Municipal de Saúde (SEMUSA) de Porto Velho/RO, para compreensão e aceite do estudo na instituição. No intuito de familiarizar-se com o ambiente, a pesquisadora participou na condição de observadora, de atividades do cotidiano da instituição, como consultas médicas, grupos terapêuticos e triagem.

Foram feitas consultas nos prontuários dos pacientes e foram selecionados todos aqueles que atendiam aos critérios de inclusão do estudo. A partir disso, foram marcados encontros no CAPSad com alguns dos pacientes pelo número de telefone que constavam nos prontuários de cada um. Outros foram abordados na sala de espera do CAPsad, após a realização da triagem. Os primeiros 26 pacientes entrevistados foram alocados para o G1=Grupo Experimental e os outros 26 foram direcionados para o G2=Grupo Controle. No contato inicial a pesquisadora se apresentou aos pacientes alocados e, posteriormente falou dos objetivos e outras partes do projeto.

Para todos aqueles que aceitaram participar do estudo, foi apresentado o Termo de Consentimento Livre e Esclarecido (TCLE). Uma vez assinado o TCLE, os participantes começaram a responder aos questionários, que teve duração de aproximadamente 40 minutos, acompanhada pela pesquisadora que supervisou todo o processo de coleta sem qualquer interferência, numa sala restrita a este trabalho.

Após a finalização da aplicação dos questionários, os pacientes foram convidados a participarem da avaliação da quantidade de nicotina presente nos pulmões (CO2) por meio do Monoxímetro, realizada pela própria pesquisadora do projeto.

O G2 recebeu o tratamento tradicional que é oferecido pelo CAPSad. Trata-se de um programa oferecido pelo Ministério da Saúde em parceria com o Instituto Nacional do Câncer (INCA) para as unidades de saúde que trabalham no tratamento da dependência química no Brasil. Este programa de tratamento tem como base a Terapia Cognitivo Comportamental (TCC), constituída em quatro sessões, com encontros semanais, e duas sessões quinzenais, seguidas por reuniões mensais de manutenção. Cada sessão tem a durabilidade de uma hora e meia, aproximadamente.

Na primeira sessão foi realizada uma atividade de psicoeducação para que os pacientes tomassem consciência do seu problema, entendessem os malefícios do cigarro à saúde e identificasse perante breves questionários qual era o tipo de dependência que os mesmos apresentavam. Da segunda à quarta sessão foram abordados os benefícios que o fumante pode ter ao abandonar o uso do cigarro e estratégias para vencer os obstáculos e permanecer sem fumar. As sessões quinquenais de manutenção foram feitas para verificar a evolução do paciente.

No G1 utilizou-se uma adaptação do programa de Prevenção de Recaídas baseada em mindfulness para comportamento de recaídas (Bowen, Chawla, \& Marlatt, 2015). Foi desenvolvido em oito sessões, com encontros semanais, tendo uma durabilidade de uma hora e meia a duas horas no máximo. 
Nas três primeiras sessões, foi discutida a importância da consciência do papel do piloto automático na recaída, nas sessões quatro a seis reforçaram-se as práticas das sessões anteriores, também se identificaram perfis individuais de risco e foram ensinadas práticas de enfrentamento a essas situações de risco. Finalmente, nas sessões sete e oito foram trabalhadas práticas para a manutenção de estilos de vida que sustente tanto a recuperação quanto a prática de mindfulness (Tabela 1).

Tabela 1. Apresentação das oito sessões de mindfulness que foram desenvolvidas nos usuários do G2=experimental.

\begin{tabular}{|c|c|c|}
\hline Sessão & Conteúdo Teórico & Práticas \\
\hline \multirow{3}{*}{$\begin{array}{l}\text { Sessão } 1 \\
\text { Piloto automático e } \\
\text { recaída. }\end{array}$} & \multirow{3}{*}{ Apresentação e objetivos. } & Escaneamento corporal em seis de sete dias. \\
\hline & & Mindfulness em uma atividade diária. \\
\hline & & Planilha de monitoramento de prática diária. \\
\hline \multirow{3}{*}{$\begin{array}{l}\text { Sessão } 2 \\
\text { Piloto automático e } \\
\text { recaída. }\end{array}$} & \multirow{3}{*}{$\begin{array}{l}\text { Consciência do papel } \\
\text { do piloto automático na } \\
\text { recaída. }\end{array}$} & Escaneamento corporal em seis de sete dias. \\
\hline & & Mindfulness em uma atividade diária. \\
\hline & & Planilha de monitoramento de prática diária. \\
\hline \multirow{3}{*}{$\begin{array}{l}\text { Sessão } 3 \\
\text { Piloto automático e } \\
\text { recaída. }\end{array}$} & \multirow{3}{*}{$\begin{array}{l}\text { Consciência do papel } \\
\text { do piloto automático na } \\
\text { recaída. }\end{array}$} & Escaneamento corporal em seis de sete dias. \\
\hline & & Mindfulness em uma atividade diária. \\
\hline & & Planilha de monitoramento de prática diária. \\
\hline \multirow{6}{*}{$\begin{array}{l}\text { Sessão } 4 \\
\text { Mindfulness em } \\
\text { situações de alto } \\
\text { risco. }\end{array}$} & \multirow{6}{*}{$\begin{array}{l}\text { Apresentação e objetivos. } \\
\text { Reforçar as práticas das } \\
\text { sessões anteriores. } \\
\text { Identificara perfis } \\
\text { individuais de risco. }\end{array}$} & Escaneamento corporal em seis de sete dias. \\
\hline & & Mindfulness em uma atividade diária. \\
\hline & & Planilha de monitoramento de prática diária. \\
\hline & & Meditação sentada (seis de sete dias). \\
\hline & & $\begin{array}{l}\text { Meditação andando ou caminhada mindful pelo } \\
\text { menos duas vezes }\end{array}$ \\
\hline & & $\begin{array}{l}\text { Espaço PARAR para respirar (diariamente, em } \\
\text { especial em situações difíceis). }\end{array}$ \\
\hline \multirow{6}{*}{$\begin{array}{l}\text { Sessão } 5 \\
\text { Mindfulness em } \\
\text { situações de alto } \\
\text { risco. }\end{array}$} & \multirow{6}{*}{$\begin{array}{l}\text { Reforçar as práticas das } \\
\text { sessões anteriores. } \\
\text { Identificara perfis } \\
\text { individuais de risco. }\end{array}$} & Escaneamento corporal em seis de sete dias. \\
\hline & & Mindfulness em uma atividade diária. \\
\hline & & Planilha de monitoramento de prática diária. \\
\hline & & Meditação sentada (seis de sete dias). \\
\hline & & $\begin{array}{l}\text { Meditação andando ou caminhada mindful pelo } \\
\text { menos duas vezes. }\end{array}$ \\
\hline & & $\begin{array}{l}\text { Espaço PARAR para respirar (diariamente, em } \\
\text { especial em situações difíceis). }\end{array}$ \\
\hline \multirow{6}{*}{$\begin{array}{l}\text { Sessão } 6 \\
\text { Mindfulness em } \\
\text { situações de alto } \\
\text { risco. }\end{array}$} & \multirow{6}{*}{$\begin{array}{l}\text { Reforçar as práticas das } \\
\text { sessões anteriores. } \\
\text { Identificara perfis } \\
\text { individuais de risco. }\end{array}$} & Escaneamento corporal em seis de sete dias. \\
\hline & & Mindfulness em uma atividade diária. \\
\hline & & Planilha de monitoramento de prática diária. \\
\hline & & Meditação sentada (seis de sete dias). \\
\hline & & $\begin{array}{l}\text { Meditação andando ou caminhada mindful pelo } \\
\text { menos duas vezes. }\end{array}$ \\
\hline & & $\begin{array}{l}\text { Espaço PARAR para respirar (diariamente, em } \\
\text { especial em situações difíceis). }\end{array}$ \\
\hline
\end{tabular}




\begin{tabular}{|c|c|c|}
\hline Sessão & Conteúdo Teórico & Práticas \\
\hline \multirow{2}{*}{$\begin{array}{l}\text { Sessão } 7 \\
\text { Autocuidado e } \\
\text { Equilíbrio de Estilo } \\
\text { de Vida }\end{array}$} & \multirow{2}{*}{$\begin{array}{l}\text { Apresentação e objetivos } \\
\text { Manutenção de estilos } \\
\text { de vida. }\end{array}$} & $\begin{array}{l}\text { Meditação sentado: Meditação da Bondade } \\
\text { Amorosa. }\end{array}$ \\
\hline & & $\begin{array}{l}\text { Espaço PARAR para respirar (diariamente, em } \\
\text { especial em situações difíceis). }\end{array}$ \\
\hline \multirow{2}{*}{$\begin{array}{l}\text { Sessão } 8 \\
\text { Suporte Social e } \\
\text { Prática Continuada. }\end{array}$} & \multirow{2}{*}{$\begin{array}{l}\text { Apresentação e objetivos } \\
\text { Manutenção de estilos } \\
\text { de vida. }\end{array}$} & Escaneamento corporal. \\
\hline & & Meditação conclusiva. \\
\hline
\end{tabular}

Nota: Adaptação do programa de recaída publicado por Bowen, S., Chawla, N., \& Marlatt, A. G. (2015). Prevenção de recaída baseada em mindfulness para comportamentos aditivos: Um guia para o clínico. Rio de Janeiro. Editora Cognitiva.

\section{Tratamento Estatístico}

Para análise e processamento dos dados utilizou-se o programa IBM SPSS Statistics 20. Para verificar a curva de normalidade dos dados foi realizado o teste de Shapiro-Wilk ( $p>0.05)$. Para os dados categorizados foram utilizadas as frequências absolutas e relativas. Para análise das prevalências e comparação de proporções entre os grupos em cada momento, utilizou-se o teste qui-quadrado. 0 teste de McNemar foi utilizado para comparar as proporções pré e pós-intervenção das variáveis, ansiedade, depressão e o monóxido de carbono (CO2) dos participantes dentro de cada grupo. Foi considerado para as diferenças o valor de $p<0,05$.

\section{Resultados}

\section{Questionário Sociodemográfico e Escala Fagerström}

A amostra foi composta por um total de 52 participantes com Média de Idade (MI) de 48,61 \pm 10,29, sendo 26 do grupo experimental $(M I=48,31 \pm 9,29)$ e 26 do grupo controle $(\mathrm{Ml}=48,92 \pm 11,37)$. Os maiores valores representados pelas frequências (\%) foram na idade de 20 a 59 anos (73,1\%), sexo feminino (82,7\%), escolaridade: ensino fundamental (44,2\%), classe sócio- econômica média baixa (classe c) $(69,2 \%)$, estado civil solteiro (65,4\%) e na Escala Fagerström: elevado (42,5\%) (Tabela 2).

Na comparação entre o grupo experimental e controle, nas variáveis sociodemográficas e a Escala Fagerström, não apresentaram diferenças significativas $(p>0,05)$ (Tabela 2).

Todos os tabagistas fumavam exclusivamente cigarro, isto é, não foi encontrado ninguém que fumasse somente charuto, palheiro ou cachimbo nessa amostra.

\section{Inventário de Beck de Depressão, de Ansiedade (BDI e BAI) e o Monoxímetro}

$\mathrm{Na}$ análise da pré-intervenção, conforme mostra a Tabela 3, através da análise de prevalência, foi possível perceber que, os grupos: experimental e controle, não apresentaram diferença significativa de associação entre eles, em nenhuma das variáveis de estudo: ansiedade, depressão e níveis de monóxido de carbono nos pulmões $(p>0,05)$, ou seja, ambos apresentaram características semelhantes. 
Tabela 2. Representação dos Dados Sociodemográficos e da Escala Fagerström pelas prevalências \% (n) dos grupos experimental e controle dos pacientes fumantes em tratamento no Centro de Atenção Psicossocial de álcool e drogas. Porto Velho/RO, 2018.

\begin{tabular}{|c|c|c|c|c|c|}
\hline Variáveis & Total & & Experimental* & Controle* & Valor-p \\
\hline Idade & $n$ & $\%$ & $\%(n)$ & $\%(n)$ & 0,203 \\
\hline 20 a 59 anos & 38 & 73,1 & $52,6(20)$ & $47,4(18)$ & \\
\hline Maior ou igual 60 anos & 14 & 26,9 & $42,9(6)$ & $57,1(8)$ & \\
\hline Sexo & & & & & 0,267 \\
\hline Feminino & 43 & 82,7 & $51,2(22)$ & $48,8(21)$ & \\
\hline Masculino & 9 & 17,3 & $44,4(4)$ & $55,6(5)$ & \\
\hline Escolaridade & & & & & 0,324 \\
\hline Analfabeto & 5 & 9,6 & $60,0(3)$ & $40,0(2)$ & \\
\hline Ensino Fundamental & 23 & 44,2 & 39,1 (9) & $60,9(14)$ & \\
\hline Ensino Médio & 17 & 32,7 & $58,8(10)$ & $41,2(7)$ & \\
\hline Superior & 7 & 13,5 & $57,1(4)$ & $42,9(3)$ & \\
\hline Renda & & & & & 0,601 \\
\hline Classe B & 8 & 15,4 & $50,0(4)$ & $50,0(4)$ & \\
\hline Classe C & 36 & 69,2 & $50,0(18)$ & $50,0(18)$ & \\
\hline Classe D-E & 8 & 15,4 & $50,0(4)$ & $50,0(4)$ & \\
\hline Estado Civil & & & & & 0,194 \\
\hline Solteiro & 34 & 65,4 & $47,1(16)$ & $52,9(18)$ & \\
\hline Casado-União Estável & 18 & 34,6 & $55,6(10)$ & $44,5(8)$ & \\
\hline Fagerström & & & & & 0,331 \\
\hline Baixo & 20 & 38,5 & $50,0(3)$ & $50,0(3)$ & \\
\hline Médio & 10 & 19,2 & $46,9(15)$ & $53,1(17)$ & \\
\hline Elevado & 22 & 42,5 & $57,1(8)$ & $42,9(6)$ & \\
\hline
\end{tabular}

Nota: Teste qui-quadrado $*(p>0,05)$

Tabela 3. Análises de prevalências \% (n) entre os grupos experimental e controle em dois momentos pré e pós-teste nos Inventários de Depressão e Ansiedade (BDI e BAI) e no Monoxímetro dos pacientes fumantes em tratamento no Centro de Atenção Psicossocial de álcool e drogas. Porto Velho/RO, 2018.

\begin{tabular}{|c|c|c|c|c|c|c|c|c|}
\hline \multirow{3}{*}{ Variáveis } & \multicolumn{4}{|c|}{ Pré-intervenção } & \multicolumn{2}{|c|}{ Pós-intervenção } & \multicolumn{2}{|c|}{ Valor-p } \\
\hline & & Experimental & Controle & & Experimental & Controle & 1 & ? \\
\hline & $n$ & $\%(n)$ & $\%(n)$ & $n$ & $\%(n)$ & $\%(n)$ & 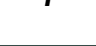 & 2 \\
\hline Ansiedade & & & & & & & 0,731 & 0,043 \\
\hline Sem & 7 & $42,9(3)$ & $57,1(4)$ & 24 & $70,8(17)$ & $29,2(7)$ & & \\
\hline Leve & 18 & $55,6(10)$ & $44,4(8)$ & 12 & $25,0(3)$ & $75,5(9)$ & & \\
\hline Moderada & 10 & $60,0(6)$ & $40,0(4)$ & 8 & $37,5(3)$ & $62,5(5)$ & & \\
\hline Severa & 17 & $41,2(7)$ & $58,8(10)$ & 8 & $37,5(3)$ & $62,5(5)$ & & \\
\hline Depressão & & & & & & & 0,974 & 0,33 \\
\hline Sem & 7 & $57,1(4)$ & $42,9(3)$ & 19 & $68,4(13)$ & $31,6(6)$ & & \\
\hline Leve & 26 & $50,0(13)$ & $50,0(13)$ & 14 & $28,6(4)$ & $1,4(10)$ & & \\
\hline Moderada & 6 & $50,0(3)$ & $50,0(3)$ & 9 & $44,4(4)$ & $55,6(5)$ & & \\
\hline Severa & 13 & $46,2(6)$ & $53,8(7)$ & 10 & $50,0(5)$ & $50,0(5)$ & & \\
\hline
\end{tabular}




\begin{tabular}{|c|c|c|c|c|c|c|c|c|}
\hline \multirow{3}{*}{ Variáveis } & \multicolumn{4}{|c|}{ Pré-intervenção } & \multicolumn{2}{|c|}{ Pós-intervenção } & \multicolumn{2}{|c|}{ Valor-p } \\
\hline & & Experimental & Controle & & Experimental & Controle & 1 & ? \\
\hline & $n$ & $\%(n)$ & $\%(n)$ & $n$ & $\%(n)$ & $\%(n)$ & 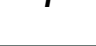 & \\
\hline Monox. CO2 & & & & & & & 0,814 & 0,033 \\
\hline Ex-fumante & 6 & $50,0(3)$ & $50,0(3)$ & 24 & $75,0(18)$ & $25,0(6)$ & & \\
\hline Fumante & 32 & $46,9(15)$ & $53,1(17)$ & 21 & $23,8(5)$ & $76,2(16)$ & & \\
\hline $\begin{array}{c}\text { Fumante } \\
\text { Dependente }\end{array}$ & 14 & $57,1(8)$ & $42,9(6)$ & 7 & $42,9(3)$ & $57,1(4)$ & & \\
\hline
\end{tabular}

Nota: Pré-intervenção = características semelhantes (únicas) entre os grupos experimental e controle no pré-teste; Valores p 1=Pré-intervenção valor $\mathrm{p} 2$ = Pós-intervenção; teste Qui-quadrado $p<0,05$; Monox.CO2=monóxido de carbono

Na análise pós-intervenção, os grupos experimental e controle mostraram diferenças significativas na variável ansiedade e nos níveis de monóxido de carbono nos pulmões. Sendo que, a ansiedade nas categorias "sem ansiedade" aumentou do Pré para o Pós-intervenção, e a situação inversa ocorreu para as demais categorias, ou seja, houve uma diminuição na ansiedade leve, moderada e severa. Na avaliação do CO2 pelo Monoxímetro a presença de CO2 nos pulmões diminuiu no fumante e fumante dependente.

Na variável depressão, tanto no momento Pré e Pós-intervenção entre os grupos não ocorreu diferença significativa. Observa-se que no pós-intervenção ocorreu o aumento da categoria "sem depressão", e diminuição nas categorias leve e moderada, mas sem significância.

$\mathrm{Na}$ Tabela 4 mostra a frequência de ansiedade, depressão e níveis de monóxido de carbono nos pulmões categorizados por: presença de ansiedade, depressão ou CO2 e ausência de ansiedade, depressão ou CO2; separados por grupos experimental e controle; pré-intervenção e pós-intervenção. Na pós-intervenção no grupo experimental, ocorreu modificações significativas, havendo diminuição nas categorias "presença da ansiedade" e "presença de depressão", já nas categorias "ausência da ansiedade" e "ausência de depressão" houve um aumento. Nas variáveis "presença ou ausência de CO2" os resultados demonstraram redução de pacientes com "presença de CO2", considerados fumantes e aumento de pacientes com "ausência de C02", considerados não-fumantes. Na pós-intervenção do grupo controle nas variáveis presença ou ausência de ansiedade, depressão e CO2 a análise estatística demonstrou não haver mudanças significativas conforme mostra a Tabela 4.

Tabela 4. Dados da frequência de ansiedade, depressão e níveis de monóxido de carbono nos pulmões em relação aos grupos experimental, controle e a pré-intervenção e pós-intervenção dos pacientes fumantes em tratamento no Centro de Atenção Psicossocial de álcool e drogas. Porto Velho/RO, 2018.

\begin{tabular}{|c|c|c|c|c|c|}
\hline \multirow[b]{2}{*}{ Grupos } & \multicolumn{2}{|c|}{ Pré-intervenção } & \multicolumn{2}{|c|}{ Pós-intervenção } & \multirow{2}{*}{$\begin{array}{c}\text { Valor } \\
\quad p\end{array}$} \\
\hline & $\begin{array}{c}\text { Presença } \\
\text { ansiedade } n(\%)\end{array}$ & $\begin{array}{c}\text { Ausência } \\
\text { ansiedade } n(\%)\end{array}$ & $\begin{array}{c}\text { Presença } \\
\text { ansiedade } n(\%)\end{array}$ & $\begin{array}{c}\text { Ausência } \\
\text { ansiedade } n(\%)\end{array}$ & \\
\hline Experimental & $8(30,8)$ & $2(7,7)$ & $1(3,8)$ & $15(57,7)$ & $<0,001$ \\
\hline \multirow[t]{2}{*}{ Controle } & $1(3,8)$ & $3(11,5)$ & $18(69,2)$ & $4(15,4)$ & 0,375 \\
\hline & $\begin{array}{l}\text { Presença } \\
\text { depressão }\end{array}$ & $\begin{array}{l}\text { Ausência } \\
\text { depressão }\end{array}$ & $\begin{array}{l}\text { Presença } \\
\text { depressão }\end{array}$ & $\begin{array}{l}\text { Ausência } \\
\text { depressão }\end{array}$ & \\
\hline Experimental & $10(38,5)$ & $3(11,5)$ & $1(3,8)$ & $12(46,2)$ & 0,012 \\
\hline
\end{tabular}


Pág 139

\begin{tabular}{|c|c|c|c|c|c|}
\hline \multirow[b]{2}{*}{ Grupos } & \multicolumn{2}{|c|}{ Pré-intervenção } & \multicolumn{2}{|c|}{ Pós-intervenção } & \multirow{2}{*}{$\begin{array}{c}\text { Valor } \\
p\end{array}$} \\
\hline & $\begin{array}{c}\text { Presença } \\
\text { ansiedade } n(\%)\end{array}$ & $\begin{array}{c}\text { Ausência } \\
\text { ansiedade } n(\%)\end{array}$ & $\begin{array}{c}\text { Presença } \\
\text { ansiedade } n(\%)\end{array}$ & $\begin{array}{c}\text { Ausência } \\
\text { ansiedade } n(\%)\end{array}$ & \\
\hline \multirow[t]{3}{*}{ Controle } & $2(7,7)$ & $1(3,8)$ & $5(19,2)$ & $18(69,2)$ & 0,453 \\
\hline & Presença & \multirow{2}{*}{ Ausência CO2 } & \multirow{2}{*}{$\begin{array}{l}\text { Presença } \\
\text { CO2 }\end{array}$} & Ausência & \\
\hline & $\mathrm{CO} 2$ & & & $\mathrm{CO} 2$ & \\
\hline Experimental & $16(61,5)$ & $2(7,7)$ & $1(3,8)$ & $7(26,9)$ & $<0,001$ \\
\hline Controle & $5(19,2)$ & $1(3,8)$ & $18(69,2)$ & $2(7,7)$ & 0,453 \\
\hline
\end{tabular}

Nota: Teste McNemar (teste de frequência/proporções antes e depois do experimento); Presença de CO2=fumante; Ausência de CO2=ex-fumante

Os achados principais do presente estudo foram diminuição dos sintomas de ansiedade $(p=0,001)$, depressão $(p=0,012)$ e redução dos níveis de monóxido de carbono nos pulmões $(p=0,001)$ dos pacientes do grupo experimento pós-intervenção apresentados na Tabela 4.

\section{Discussão}

Nesse estudo pode-se verificar que o programa de tratamento baseado em Mindfulness para tabagistas teve efeitos positivos observados nos níveis reduzidos de monóxido de carbono nos seus pulmões e diminuição dos sintomas de ansiedade e depressão.

Nesse estudo pode-se verificar que o programa de tratamento baseado em Mindfulness para tabagistas teve efeitos positivos observados nos níveis reduzidos de monóxido de carbono nos seus pulmões e diminuição dos sintomas de ansiedade e depressão.
Ressalta-se que a ansiedade e depressão são emoções inerentes ao ser humano, e que a experiência de medo ou ansiedade não causa um transtorno de ansiedade, assim como, a tristeza não causa transtorno depressivo, mas sim a maneira como o indivíduo reage a esses sintomas, que exacerbam sua intensidade e duração, causando sofrimento e interferindo na qualidade de vida (Germer, Siegel, \& Fulton, 2016).

Como citado no decorrer do texto, a ansiedade e a depressão são sintomas comuns entre pacientes tabagistas que podem se somar a outros fatores, como a fissura (intenso desejo de usar a droga) e síndrome de abstinência e dessa forma influenciar no processo de recaída dificultando a recuperação (Heppener \& Spears, 2016).

Para enfrentar essas situações, os pacientes muitas vezes agem de maneira automática, e para aliviar os sintomas da fissura, ou da síndrome de abstinência acabam se automedicando, ou usando o cigarro como forma de aliviar tais sintomas. No entanto, a sensação de alívio é momentânea. A utilização do mindfulness atua como medida de prevenção para o alívio de sintomas, pois esta prática faz com que o paciente entenda e aceite o que está sentindo em vez de se esquivar da situação que acentuaria esses sintomas (Mouzinho et al., 2018).

A partir das tradições budistas e comportamentais, as terapias baseadas em mindfulness e aceitação, foram desenvolvidas para ajudar as pessoas a aprender novas formas de respostas a fim de reduzir os sintomas de ansiedade e melhorar a qualidade de vida. Esta prática faz com que o paciente haja com suas experiências de maneira mais ampla, em vez de estreita, mais compassiva, menos julgadora e descentrada. Dessa forma, consegue perceber os pensamentos de forma objetiva, e não como verdade absoluta. Com isto, o paciente passa a envolver-se em ações que sejam importantes para si, em vez de habitualmente evitá-las (Germer, Siegel, \& Fulton, 2016). 
Pág 140

Morón, Rey e Martí (2014) explicam que um dos objetivos desta prática é ajudar ao paciente a reconhecer e se preparar para situações de alto risco de consumo. Também é enfatizada a aceitação no lugar da culpa e da vergonha pelo desejo de usar a droga. Portanto o treinamento de mindfulness serve para observar as experiências de maneira consciente e sem julgamento, o que faz com que o paciente seja mais consciente de seus estados emocionais e fisiológicos, e desta maneira seja capaz de identificar os fatores internos que ativam a recaída.
Uma das intenções desta prática é fazer com que as pessoas aprendam a cultivar uma resposta atenta, compassiva e receptiva às experiências. Por meio desta prática é possível voltar-se de forma gentil aos sentimentos e sensações ansiosas, com bondade e compaixão, enquanto se mantém o foco no momento presente, mesmo que por vezes a atenção se desvie para acontecimentos passados ou futuros. Esta atitude pode ajudar a neutralizar respostas ansiosas aprendidas e a promover uma vida mais satisfatória (Vinci et al., 2016).

Morón, Rey e Martí (2014) explicam que um dos objetivos desta prática é ajudar ao paciente a reconhecer e se preparar para situações de alto risco de consumo. Também é enfatizada a aceitação no lugar da culpa e da vergonha pelo desejo de usar a droga. Portanto o treinamento de mindfulness serve para observar as experiências de maneira consciente e sem julgamento, o que faz com que o paciente seja mais consciente de seus estados emocionais e fisiológicos, e desta maneira seja capaz de identificar os fatores internos que ativam a recaída.

Um estudo feito por Davis et al. (2014) de ensaio clínico randomizado realizado nos Estados Unidos nos anos 2011 e 2012 com 175 sujeitos, demonstrou que o mindfulness teve efeitos positivos para a cessação do tabagismo, havendo taxas de abstinência em até 24 semanas após o fim do tratamento (Zeidan et al., 2014). Assim como outros achados no mesmo estudo mostraram diminuição de sintomas de depressão e ansiedade.

Este estudo é um exemplo da eficácia do mindfulness no tratamento de sintomas ansiosos pacientes em tratamento para o tabagismo. Os dados da variável ansiedade demonstraram que na pré-intervenção para a pós-intervenção ouve diferença significativa. Ouve aumento da categoria "sem ansiedade", que na pré-intervenção era de (42,9\%) e na pós-intervenção foi para (70,8\%), sendo o valor de $p=0,043$.

$\mathrm{Na}$ tentativa de entender como o mindfulness contribui para o alívio da ansiedade a nível neural, pesquisadores como Zeidan, Martucci, Kraft, Mchaffie e Coghill (2014) fizeram um estudo utilizando ressonância magnética para decifrar este acontecimento. Participaram 15 sujeitos saudáveis, sem nenhuma experiência prévia de meditação, ambos participaram de quatro dias de treinamento de meditação da atenção plena. Os resultados demonstraram que a ansiedade foi significativamente reduzida em todas as sessões que os sujeitos meditaram. 0 alívio da ansiedade relacionado à meditação foi associado a ativação do córtex cingulado anterior, córtex pré-frontal ventromedial e ínsula anterior (mecanismos responsáveis pela tomada de desições, raciocínio social e controle emocional). Com este estudo os autores concluiram que, o mindfulness atenua a ansiedade através de mecanismos envolvidos na regulação dos processos de pensamento auto-referenciais.

O presente estudo também é um exemplo da eficácia da atenção plena nos sintomas depressivos. Apesar da análise dos resultados da variável depressão indicaram não haver diferença significativa entre os grupos da pré-intervenção para a pós-intervenção, constatou-se aumento da categoria "sem depressão" na pós-intervenção $(68,4 \%)$ se relacionada à pré-intervenção $(57,1 \%)$, sendo o nível de significância do grupo experimental $(p=0,974)$ e $(p=0,330)$ no grupo controle.

Estudo semelhante, foi realizado em 2016 por Vinci, Spears, Peltier e Copeland (2016) com 72 pacientes tabagistas. Tal estudo buscou examinar em que medida a atenção plena media a relação entre sintomas depressivos e tabagismo. Os autores 
Pág 141

Apesar das intervenções baseadas em mindfulness serem igualmente eficazes como outras abordagens empiricamente validadas (por exemplo, TCC, gerenciamento de contingência e outras), o mindfulness apresenta um diferencial. A técnica contribui para a ampliação da consciência do praticante sobre seus sentimentos, pensamentos e emoções. Desse modo, além de ajudar na manutenção da abstinência em pacientes dependentes, auxilia no manejo da fissura e controle de impulsos, também fomenta no praticante atitudes mais adaptativas e menos automáticas, benefíciando a saúde e bem-estar biopsicossocial daqueles que o praticam. associaram a depressão grave a sujeitos que fumavam mais, já que estes teriam baixa capacidade de julgamento dos pensamentos. Os resultados demonstraram melhor efetividade da prática do mindfulness para pacientes com sintomas depressivos leves, estes, obtiveram melhoras significativas, apresentaram maior capacidade de aceitação das emoções e dos pensamentos sem julgamento.

Em relação á depressão Germer, Siegel e Fulton (2016), afirmam que, para o tratamento da mesma, existem uma série de mecanismos responsáveis pela eficácia de tratamentos baseados em mindfulness. Segundo eles, este tipo de tratamento pode ajudar aos pacientes a enfrentar dor e sofrimento emocionais cultivando qualidades como tolerância a afeto e autoaceitação. Ao aprender enfrentar a dor diretamente, os pacientes mudam sua relação com ela. Em vez de reagir das formas habituais (odiando-as, rumindo sobre elas ou afastando-se), eles podem começar a se abrir para ela, a tornar-se curioso a seu respeito e aceitá-la de forma gradual. Paradoxalmente, tal aceitação com frequência leva a mudanças positivas.

Autores como Nunes \& Castro (2010) e Heppner \& Spears (2016) afirmam que a ausência ou diminuição de sintomas de transtornos psíquicos como a ansiedade e a depressão, podem contribuir para a cessação do tabagismo. No grupo experimento pós-intervenção, ocorreu uma diminuição nos níveis de monóxido de carbono (CO2) no pulmão da maioria dos pacientes. Alguns deles alcançaram a classificação não-fumante conforme o aparelho de medição (26,9\%). Acredita-se que, os níveis de monóxido de carbono diminuíram de forma altamente significativa suas taxas de consumo do uso do cigarro.

\section{Conclusão}

Perante os resultados deste estudo é possível afirmar que, a prática de mindfulness realizada no período de sessenta dias no CAPSad no Município de Porto Velho, estado de Rondônia, foi eficaz para evitar a recaída dos pacientes tabagistas. Houve diminuição dos sintomas de depressão e ansiedade, bem como o nível de monóxido de carbono nos pulmões. Os resultados estatísticos demonstraram que, os pacientes que participaram do programa de prevenção de recaídas baseado em mindfulness obtiveram resultados significativamente melhores na medida de $\mathrm{CO} 2$ e ansiedade se comparados ao grupo controle.

Espera-se que, este estudo incite a possibilidade de novas pesquisas ou instigue a necessidade da continuidade de estudos longitudinais com medidas a longo prazo, referente a eficácia do mindfulness para o tratamento não só do tabagismo mais de outras substâncias psicoativas, uma vez que são poucas as pesquisas sobre esta prática no Brasil e tendo em vista a relevância dos resultados deste estudo.

Apesar das intervenções baseadas em mindfulness serem igualmente eficazes como outras abordagens empiricamente validadas (por exemplo, TCC, gerenciamento de contingência e outras), o mindfulness apresenta um diferencial. A técnica contribui para a ampliação da consciência do praticante sobre seus sentimentos, pensamentos e emoções. Desse modo, além de ajudar na manutenção da abstinência em pacientes dependentes, auxilia no manejo da fissura e controle de impulsos, também fomenta no praticante atitudes mais adaptativas e menos automáticas, benefíciando a saúde e bem-estar biopsicossocial daqueles que o praticam. 


\section{Referências}

Associação Brasileira de Empresas de Pesquisa [ABEP]. (2017). Critério de classificação econômica Brasil. http://www.abep.org/criterio-brasil

Beck, J. S. (2013). Terapia Cognitivo Comportamental: Teoria e Prática. Porto Alegre: Artmed.

Beck, A. T., Ward, C. H., Mendelson, M., Mock, J., \& Erbaugh, J. (1961). An inventory for measuring depression. Archives of General Psychiatry, 4, 561-571. doi: https:// doi.org/10.1001/archpsyc.1961.01710120031004

Beck, A. T., Epstein, N., Brown, G., \& Steer, R. A. (1988). An inventory for measuring clinical anxiety. Psychometric properties. Journal of Consulting and Clinical Psychology, 6(5), 893-897. doi: https://doi.org/10.1037/0022-006X.56.6.893

Beck, A. T., \& Steer, R. A. (1993). Beck anxiety inventory (BAl) San Antonio: Phychological corporation.

Bowen, S., Chawla, N., \& Marlatt, A. G. (2015). Prevenção de recaída baseada em mindfulness para comportamentos aditivos: Um guia para o clínico. Rio de Janeiro. Editora Cognitiva.

Carmo, J. T., \& Pueyo, A. A. (2002). A adaptação do português do Fagerström test for nicotine dependence (FTND) para avaliar a dependência e tolerância à nicotina em fumantes brasileiros. Revista Brasileira de Medicina, 9(5),73-80. Recuperado de file:///C:/Users/UNIR/Downloads/A adaptacao ao portugues do Fagerstrom t.pdf

Cunha, J. A. (2001). Manual da versão em português das escalas Beck. São Paulo: Casa do Psicólogo.

Davis, J. M., Maggie, S. G., Anderson, C., Manley, A., Smith, S., \& Baker, T. (2014). Randomized Trial on Mindfulness Training for Smokers Targeted to a Disadvantaged Population. Substace Use and Misuse, 49(5), 571-585. doi: http://10.3109 / 10826084.2013 .770025

Diehl, A., Cordeiro, D. C., \& Laranjeira, R. (2011). Dependência Química, Prevenção, Tratamento e Políticas Públicas. Porto Alegre: Artmed.

Fagerström, K. O., \& Schneider, N. G. (1989). Measuring nicotine dependence: a review of the Fagerstrom Tolerance Questionnaire. Journal of Behavioral Medicine, 12(2), 159-82. doi: https://doi.org/10.1007/BF00846549

Germer, C. K., Siegel, R. D., \& Fulton, P. R. (2016). Mindfulness e Psicoterapia. (2a. ed.). Porto Alegre: Artmed.

Gomes-Oliveira, M. H., Gorenstein, C., Lotufo Neto, F., Andrade, L. H., \& Wang, Y. P. (2012). Validação da versão em português brasileiro do Beck Depression Inventory-II em uma amostra da comunidade. Revista Brasileira de Psiquiatria, 34(4), 389-394. doi: https://doi.org/10.1016/j.rbp.2012.03.005

Gorenstein, C., \& Wang, Y. P. (2016). Inventário de Depressão de Beck (BDI). Instrumentos de Avaliação em Saúde Mental. Porto Alegre, Artmed.

Heppner, W. L., \& Spears, C. A. (2016). Dispositional Mindfulness Predicts Enhanced Smoking Cessation and Smoking Lapse Recovery. Annalls of Beharioral Medicine, 50(45), 337-347. doi: https:// https://doi.org/10.1007/s12160-015-9759-3

Instituto Nacional do Câncer (INCA). (2017). In Programa Nacional de Controle do Tabagismo (PNCT). Recuperado de https://www.inca.gov.br/programa-nacional-de-controle-do-tabagismo

Marques, A. C. P. R. (2001). Consenso sobre o tratamento da dependência de nicotina. Revista Brasileira de Psiquiatria, 4(23), 200-214. doi: https://doi.org/10.1590/ S1516-44462001000400007

Marlatt, A., \& Donavan, D. M. (2009). Prevenção de Recaída: estratégias de manutenção no tratamento de comportamentos aditivos. Porto Alegre: Artmed. 
Miller, W. R., \& Rollnick, S. (2001). Entrevista Motivacional: preparando as pessoas para mudança de comportamento adictivos. Porto Alegre: Artmed.

Morón, D. A., Rey, R. A., \& Martí, A. C. I. (2014). Mindfulness y ciência: de la tradición a la modernidade. Madrid: Alianza Editorial.

Mouzinho, L., Costa, N., Alves, T., Silva, S., L. (2018). Contribuições do mindfulness às condições médicas: uma revisão de literatura. Psicologia, Saúde \& Doenças, 19 (2), 182-196. doi: http://dx.doi.org/10.15309/18psd190202

Nunes, S., \& Castro, M.R.P (Org.). (2010). Tabagismo: abordagem, prevenção e tratamento. Londrina: Eduel.

Pietrobon, R. C., Barbisan, J. N., \& Manfroi, W. C. (2002). Utilização do teste de dependência à nicotina de Fagerström como um instrumento de medida do grau de dependência. Jornal de Pneumologia, 28(4), 180-186. doi: https://doi. org/10.1590/S0102-35862002000400002

Prochaska, J. O., \& Diclemente, C. C. (1982). Transtheorical therapy: Toward a more integrative modelo f change. Psychotherapy: Theory, Research, \& Practice, 19(3), 276-288. doi: https:// https://doi.org/10.1037/h0088437

Rondinao, R. C., Gorayeb, R., \& Botelho, C. (2007). Características psicológicas associadas ao comportamento de fumar tabaco. Jornal Brasileiro de Pneumologia, 5(33), 592-601. doi: https://doi.org/10.1590/S1806-37132007000500016

Reitsma, M. B., \& Fullman, N. (2017). Smoking prevalence and attributable disease burden in 195 countries and territories, 1990-2015: a systematic analysis from the Global Burden of Disease Study 2015. Lancet, 389(10082), 1885-1906. doi: https// 10.1016/S0140-6736 (17) 30819-X

Vinci, C., Spears, C. A., Peltier, M. R., \& Copeland, A. L. (2016). Facets of Mindfulness Mediate the Relationship Between Depressive Symptoms and Smoking Behavior. Mindfulness. Rev.Scan, 5(7), 1408-1415. doi: https://doi.org/10.1007/s12671-016$\underline{0582-0}$

Weiss, I., \& Noto, A. R. (2017). In Neufeld e Rangé. Terapia cognitivo comportamental em grupos: das evidências à prática. Porto Alegre: Artmed.

Wenzel, A., Brown, G. K., \& Beck, A. T. (2010). Terapia cognitivo-comportamental para pacientes suicidas. Porto Alegre: Artmed.

World Health Organization (WHO). (2018). The top 10 causes of death. Recuperado em https://www.who.int/news-room/fact-sheets/detail/the-top-10-causes-of-death

Zeidan, F., Martucci, K. T., Kraft, R. A., Mchaffie, J. G., \& Coghill R. C. (2014). Neural correlates of mindfulness meditation-related anxiety relief. Social Cognitive and Affective Neurosciense 9(7), 751-759. doi: https://doi.org/10.1093/scan/nst041 